Published as: Science. 2013 March 15; 339(6125): 1284-1285.

\title{
The Brain Activity Map
}

A. Paul Alivisatos ${ }^{1,{ }^{*}}$, Miyoung Chun ${ }^{2}$, George M. Church ${ }^{3}$, Karl Deisseroth ${ }^{4}$, John P. Donoghue $^{5}$, Ralph J. Greenspan ${ }^{6}$, Paul L. McEuen ${ }^{7}$, Michael L. Roukes ${ }^{8}$, Terrence J. Sejnowski ${ }^{9,},{ }^{,}$, Paul S. Weiss ${ }^{10}$, and Rafael Yuste ${ }^{11,{ }^{*}}$

${ }^{1}$ Materials Science Division, Lawrence Berkeley National Laboratory and Department of Chemistry, University of California, Berkeley, CA 94720, USA

${ }^{2}$ The Kavli Foundation, Oxnard, CA 93030, USA

${ }^{3}$ Department of Genetics, Harvard Medical School and Wyss Institute for Biologically Inspired Engineering, Boston, MA 02115, USA

${ }^{4}$ Howard Hughes Medical Institute (HHMI) and Departments of Bioengineering and Psychiatry, Stanford University, Stanford, CA 94305, USA

${ }^{5}$ Rehabilitation Research and Development Service, Veteran Affairs Medical Center, Providence, RI 02908, USA and Brown Institute of Brain Science, Department of Neuroscience and School of Engineering, Brown University, Providence, RI 02912, USA

${ }^{6}$ Kavli Institute for Brain and Mind, University of California, San Diego, La Jolla, CA 92093, USA

${ }^{7}$ Kavli Institute at Cornell for Nanoscale Science, Department of Physics, Cornell University, Ithaca, NY 14850, USA

${ }^{8}$ Kavli Nanoscience Institute and Departments of Physics, Applied Physics, and Bioengineering, California Institute of Technology, Pasadena, CA 91125, USA

${ }^{9} \mathrm{HHMI}$, Computational Neurobiology Laboratory, Salk Institute, La Jolla, CA 92037, USA and Division of Biological Sciences, University of California, San Diego, La Jolla, CA 92093, USA

${ }^{10}$ California NanoSystems Institute, Department of Chemistry and Biochemistry, and Department of Materials Science and Engineering, University of California at Los Angeles, Los Angeles, CA 90095, USA

${ }^{11} \mathrm{HHMI}$, Departments of Biological Sciences and Neuroscience, Kavli Institute for Brain Science, Columbia University, New York, NY 10027, USA

\section{Abstract}

Neuroscientists have made impressive advances in understanding the microscale function of single neurons and the macroscale activity of the human brain. One can probe molecular and biophysical aspects of individual neurons and also view the human brain in action with magnetic resonance imaging (MRI) or magnetoencephalography (MEG). However, the mechanisms of perception, cognition, and action remain mysterious because they emerge from the real-time interactions of large sets of neurons in densely interconnected, widespread neural circuits.

It is time for a large-scale effort in neuroscience to create and apply a new generation of tools to enable the functional mapping and control of neural activity in brains with cellular and millisecond resolution. This initiative, the Brain Activity Map (BAM), could put neuroscientists in a position to understand how the brain produces perception, action,

\footnotetext{
“Corresponding author. alivis@ berkeley.edu (A.P.A.); terry@salk.edu (T.J.S.); rafaelyuste@ columbia.edu (R.Y.).
} 
memories, thoughts, and consciousness and be a major step toward a complete understanding of brain function and dysfunction. The BAM project will seek to fill the gap in our knowledge of brain activity at the circuit level, a scale between single-neuron and whole-brain function (1-3). It will provide a bridge that will enable recording and manipulating the activity of circuits, networks, and possibly eventually whole brains with single-neuron precision.

The BAM project is essentially a technology-building research program with three goals: (i) to build new classes of tools that can simultaneously image or record the individual activity of most, or even all, neurons in a brain circuit, including those containing millions of neurons; (ii) to create tools to influence the activity of every neuron individually in these circuits, because testing function requires intervention; and (iii) to understand circuit function. The third goal will require developing methods for storing, managing, and sharing large-scale imaging and physiology data, as well as developing methods for analyzing data and modeling underlying neuronal circuits, leading to emergent principles of brain function. It will be carried out by providing access for all investigators to the methods and data needed for developing, testing, and verifying theories of how the brain operates.

The BAM project will be timely; we perceive a critical coalescence of technologies from diverse fields. Model organisms could be a cornerstone of this project, as a means to extend these technologies to the human brain in a minimally invasive fashion. Invertebrates such as the worm, fly, or leech are ideal for testing new technologies, where the results can be compared to extensive, growing bodies of data on the functions of identified neurons and smaller-scale circuits. They could also be used to spearhead new capabilities for data acquisition and analysis and for theory development. Small vertebrates, such as the zebrafish, mouse, and rat, may permit scaling up of new technologies to achieve increasingly greater depth, temporal resolution, chemical sensitivity, and number of recorded neurons. With advances in technology, as yet unexplored systems could also become accessible. Finally, from the outset, we will seek to develop techniques to perform related measurements and controls in human scientific or clinical applications.

We envision the BAM project as an open, international collaboration of scientists, engineers, and theoreticians, throughout academia and industry, with work carried out both by individual laboratories and in new collective efforts. Within 5 years, it should be possible to monitor and/or to control tens of thousands of neurons, and by year 10 that number will increase at least 10 -fold. By year 15, observing 1 million neurons with markedly reduced invasiveness should be possible. With 1 million neurons, scientists will be able to evaluate the function of the entire brain of the zebrafish or areas from the cerebral cortex of the mouse or primate, for example.

In parallel, we envision developing nanoscale neural probes that can locally acquire, process, and store accumulated data. Networks of "intelligent" nanosystems would be capable of providing specific responses to externally applied signals, or to their own readings of brain activity. These, together with noninvasive optical methods, could have clinical applications for diagnosing or treating neuropsychiatric disorders, and restoring lost functions after stroke, as well as helping to generate theories of human cognition and behavior and brain disease at a neural network scale of explanation.

Many of the most devastating human brain disorders, such as epilepsy, depression, schizophrenia, autism, and dementia, may emerge when large-scale interactions within the brain are disrupted. Similarly, voluntary movements are lost when strokes, cerebral palsy, amyotrophic lateral sclerosis, or spinal cord injury disconnect the brain and body. We believe that tools and knowledge created by the BAM project may lead to new approaches to 
rebalance disordered networks and treat such diseases. Early studies have already shown that an individual can overcome profound depression when deep brain stimulation modulates disrupted neural circuits (4), and emerging brain-computer interfaces allow a person completely paralyzed from a stroke to feed themselves using a robotic arm controlled by their thoughts (5).

The BAM project will generate a trove of techniques for the neuroscience community. Just as better sequencing methods arose as a result of the Human Genome Project, concerted technology development will likely make imaging, electrophysiological, and computational techniques more powerful, more robust, and less expensive, thus supporting future neuroscience research and create clinical and commercial applications.

This proposal is meant to stimulate discussion and debate among scientists and administrators; our role is merely to help catalyze action. We believe this initiative should be funded by a partnership between federal and private organizations. It is essential that those funds not be taken away from existing neuroscience initiatives, which we view as crucial. Also, data from the BAM project should be made immediately public and accessible to all researchers, with oversight on ethical, legal, social, and safety issues.

In addition to the fundamental, clinical, and technological advances described, the BAM project will also provide fertile ground for the training of new generations of interdisciplinary researchers, equally at home in the neurosciences, the physical sciences, and engineering. The economic activities galvanized by the BAM project are expected to be comparable to those of the Human Genome Project, in which a $\$ 3.8$ billion investment generated $\$ 800$ billion in economic impact (6). We believe that when devoted and passionate groups of people join together to achieve these extraordinary goals, they will have transformational benefits for humanity.

\section{Acknowledgments}

We thank all participants in BAM workshops for input and support.

\section{References and Notes}

1. Alivisatos AP, et al. Neuron. 2012; 74:970. [PubMed: 22726828]

2. Andrews AM, Weiss PS. ACS Nano. 2012; 6:8463. [PubMed: 23088783]

3. Koch C, Reid RC. Nature. 2012; 483:397. [PubMed: 22437592]

4. Lozano AM, et al. J. Neurosurg. 2012; 116:315. [PubMed: 22098195]

5. Hochberg LR, et al. Nature. 2012; 485:372. [PubMed: 22596161]

6. Battelle, Economic impact of the human genome project. 2011 battelle.org/publications/ humangenomeproject.pdf. 


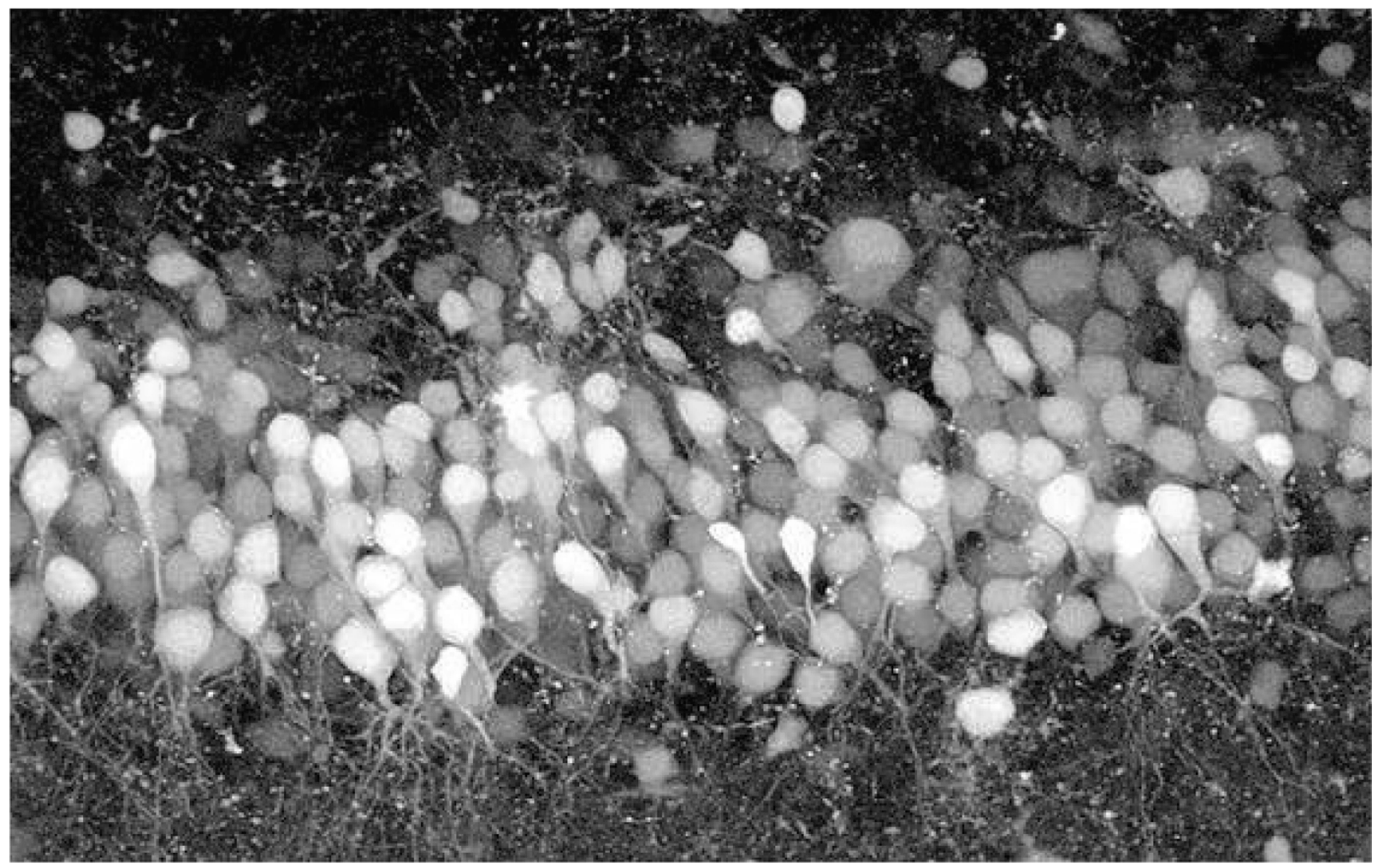

Figure. Neuronal activity in the hippocampus

Activity is shown by two-photon calcium imaging. 